\title{
Culprit Vessel Only versus Multivessel Percutaneous Coronary Intervention in Patients Presenting with ST- Segment Elevation Myocardial Infarction and Multivessel Disease
}

\author{
Dongfeng Zhang, Xiantao Song*, Shuzheng Lv, Fei Yuan, Feng Xu, Min Zhang, Wei Li, Shuai Yan
}

Department of Cardiology, Capital Medical University affiliated Beijing Anzhen Hospital, Beijing Institute of Heart, Lung and Blood Vessel Disease, Beijing, China

\begin{abstract}
Background: The best strategy for ST-segment elevation myocardial infarction (STEMI) patients with multivessel disease (MVD), who underwent primary percutaneous coronary intervention ( $\mathrm{PCI}$ ) in the acute phase, is not well established.
\end{abstract}

Objectives: Our goal was to conduct a meta-analysis comparing culprit vessel only percutaneous coronary intervention (culprit PCl) with multivessel percutaneous coronary intervention (MV-PCl) for treatment of patients with STEMI and MVD.

Methods: Pubmed, Elsevier, Embase, and China National Knowledge Infrastructure (CNKI) databases were systematically searched for randomized and nonrandomized studies comparing culprit $\mathrm{PCl}$ and $\mathrm{MV}-\mathrm{PCl}$ strategies during the index procedure. A meta-analysis was performed using Review Manager 5.1 (Cochrane Center, Denmark).

Results: Four randomized and fourteen nonrandomized studies involving 39,390 patients were included. MV-PCI strategy is associated with an increased short-term mortality (OR: $0.50,95 \% \mathrm{Cl}: 0.32$ to $0.77, p=0.002)$, long-term mortality (OR: 0.52 , $95 \% \mathrm{Cl}: 0.36$ to $0.74, \mathrm{p}<0.001)$, and risk of renal dysfunction (OR: $0.77,95 \% \mathrm{Cl}: 0.61$ to $0.97, \mathrm{p}=0.03$ ) compared with culprit $\mathrm{PCl}$ strategy, while it reduced the incidence of revascularization (OR: $2.65,95 \% \mathrm{Cl}: 1.80$ to $3.90, \mathrm{p}<0.001$ ).

Conclusions: This meta-analysis supports current guidelines which indicate that the non-culprit vessel should not be treated during the index procedure.

Citation: Zhang D, Song X, Lv S, Yuan F, Xu F, et al. (2014) Culprit Vessel Only versus Multivessel Percutaneous Coronary Intervention in Patients Presenting with ST-Segment Elevation Myocardial Infarction and Multivessel Disease. PLoS ONE 9(3): e92316. doi:10.1371/journal.pone.0092316

Editor: Claudio Moretti, S.G.Battista Hospital, Italy

Received October 30, 2013; Accepted February 21, 2014; Published March 20, 2014

Copyright: (c) 2014 Zhang et al. This is an open-access article distributed under the terms of the Creative Commons Attribution License, which permits unrestricted use, distribution, and reproduction in any medium, provided the original author and source are credited.

Funding: State Science and Technology Support Program (No. 2011BAl11B05) http://program.most.gov.cn/. The funders had no role in study design, data collection and analysis, decision to publish, or preparation of the manuscript.

Competing Interests: The authors have declared that no competing interests exist.

*E-mail: xiantao_song@163.com

\section{Introduction}

Acute ST-segment elevation myocardial infarction (STEMI) is a huge public health burden that affects many people worldwide every year. Approximately $40 \%$ to $65 \%$ of the patients presenting with STEMI have multivessel disease (MVD), which is associated with worse clinical outcomes than single-vessel disease (SVD) [1]. Percutaneous coronary intervention (PCI) is currently the favorable reperfusion treatment of choice in patients with STEMI. However, optimal strategies for STEMI patients with MVD during the index procedure, whether to treat non-culprit vessels, are still unclear.

2012 ESC guidelines [2] recommend that primary PCI should be limited to the culprit vessel with the exception of cardiogenic shock and persistent ischemia after PCI of the supposed culprit lesion while 2011 ACGF/AHA/SCAI PCI guidelines [3] suggest that PCI should not be performed in a non-culprit vessel at the time of primary PCI in patients with STEMI without hemodynamic compromise, where the classes and levels of evidence are $\mathrm{IIaB}$ and IIIB respectively. However, these suggestions were based on some retrospective or small observational studies which did not have high evidence level. The main factors supporting these guidelines are summarized as follows: complications related to non-culprit vessel PCI, overvalued stenosis, renal insufficiency, and low success rates. The advancements in PCI technology and adjunctive pharmacotherapy have led some interventionalists to operate outside of established guidelines.

Several researches showed inconsistent outcomes. Our goal was to compare the safety and efficacy of culprit vessel only PGI (culprit PCI) and multivessel PCI (MV-PCI) during the index procedure in patients with STEMI and MVD quantitatively. Therefore, we conducted a meta-analysis of randomized and nonrandomized studies.

\section{Methods}

\section{Search Strategy}

Pubmed, Elsevier, Embase, and China National Knowledge Infrastructure (CNKI) databases were systematically searched by two independent investigators (S.Y and W.L) for all articles 


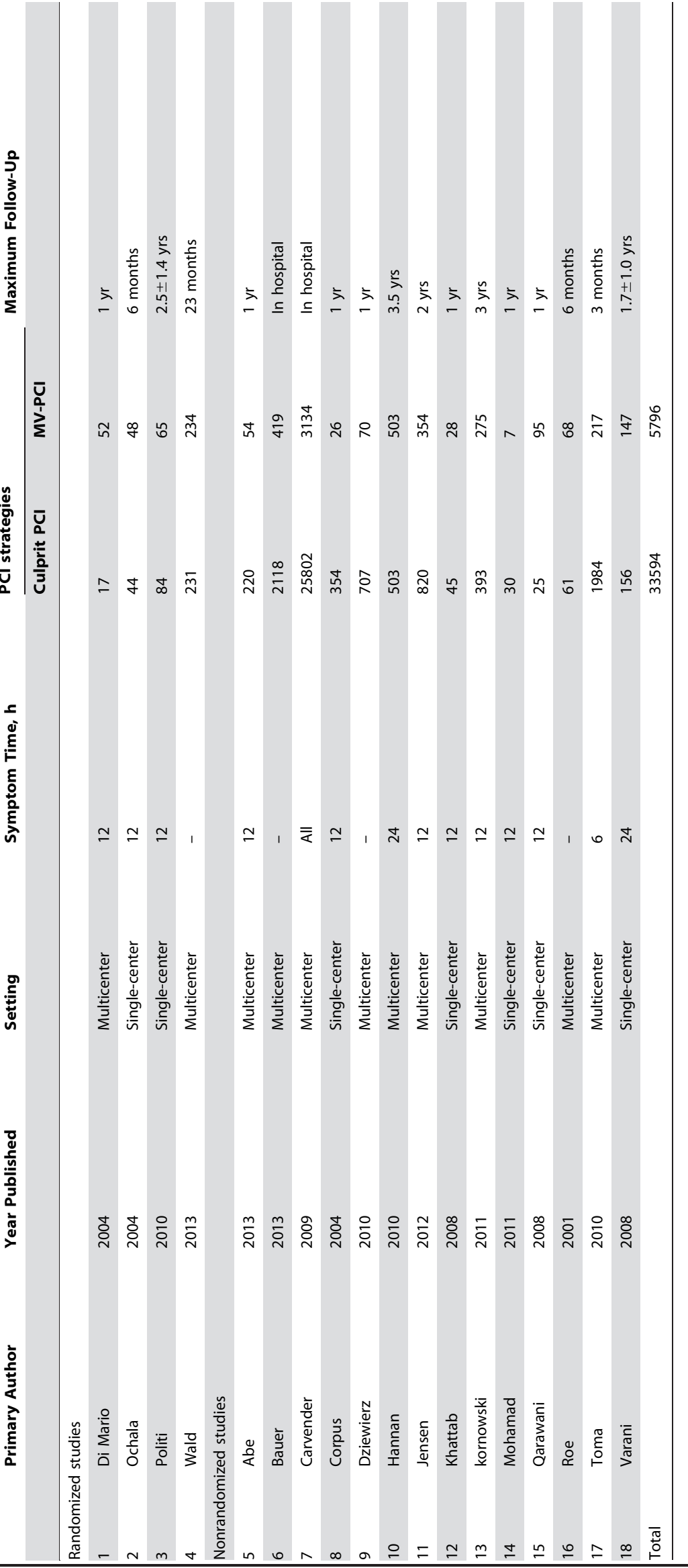

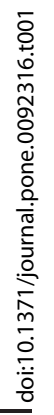


published before 6 October 2013. The following keywords were used for the search: "percutaneous coronary intervention", "STsegment elevation myocardial infarction", and "multivessel disease". Studies were excluded if they met any one of the following criteria: (1) duplicate publication, (2) ongoing or unpublished study, and (3) publication only as an abstract or as conference proceedings. References of retrieved studies were searched manually for additional potentially relevant articles. Authors of studies were contacted when results were unclear or when relevant data were not reported. Differences in investigator assessments of articles were resolved by discussing with a third investigator (D.F.Z). No language restrictions were enforced.

\section{Study Selection}

An initial screening of titles or abstracts was conducted, followed by full-text reviews. Studies' eligibility criteria included the followings: 1) a study population of STEMI patients with MVD; 2) PCI procedures included both culprit PCI and MV-PGI; 3) MV-PCI was performed during the index procedure; and 4) studies that reported quality assessment, data extraction, and endpoint data of interest. Randomized and nonrandomized studies were included. Exclusion criteria were: patient populations without concurrent STEMI and MVD, comparisons without culprit PGI or MV-PCI, and MV-PCI performed after the index procedure. Reviews, editorials, meeting abstracts, and commentaries were excluded from our analysis.

\section{Quality Assessment}

The quality of randomized studies was assessed using methods recommended by the Cochrane Collaboration based on the following six components: 1) sequence generation for allocation; 2) allocation concealment; 3) blinding of participants, personnel, and outcome assessors; 4) incomplete outcome data; 5) selective outcome reporting; and 6) other sources of bias. For nonrandomized studies, quality was assessed based on control of confounders, blinded assessment of angiography data, and preferred PCI strategy.

\section{Data Extraction}

Data were abstracted on prespecified forms by two reviewers (W.L and S.Y) that were not involved in any of the studies retrieved. Divergent assessments were resolved by discussing with a third investigator (D.F.Z). Study information was recorded as follows: study design, quality indicators, baseline clinical characteristics, and clinical outcomes.

\section{Definition and Endpoints}

The culprit PGI strategy was defined as PCI confined to culprit vessel lesions only. The MV-PCI strategy was defined as PCI in which lesions in the culprit vessel as well as $\geq 1$ nonculprit vessel lesions. All the interventions should have had taken place within the index procedure. MVD was defined as reported in each study. The primary endpoints were short-term (in hospital/30 days) and long-term mortality. Secondary endpoints included rates of renal dysfunction, reinfarction, and revascularization. Renal dysfunction as well as reinfarction and revascularization were defined as reported in each study. Mortality included both cardiac and no cardiac death.

\section{Statistical Analysis}

All statistical analysis was performed using Review Manager 5.1 (Cochrane Center, Denmark). Odds ratio (OR) and 95\% confidence intervals $(95 \%$ CI) were used as summary statistics. Heterogeneity across studies was analyzed using $\mathrm{I}^{2}\left[\mathrm{I}^{2}=(\mathrm{Q}-\mathrm{df}) / \mathrm{Q}\right.$ where $\mathrm{Q}$ is the chi-square statistic and $\mathrm{df}$ is the degree of freedom]. Values of $\mathrm{I}^{2}>50 \%$ were considered statistically significant. Pooled estimates were first calculated using the Mantel-Haenszel fixedeffects model, whereas the DerSimonian and Lair random-effects model was used if there was heterogeneity.

The following methods were used to explore sources of heterogeneity: (1) subgroup analysis (randomized and nonrandomized studies); and (2) sensitivity analysis performed by excluding trials which potentially biased meta-analysis results.

Potential publication bias was examined by visual inspection of a funnel plot. All $\mathrm{p}$ values were 2-tailed, with statistical significance set at $\mathrm{p}<0.05$. This study was performed according to the MOOSE (Meta-Analysis of Observational Studies in Epidemiology) [4] statement.

\section{Results}

Eighteen studies including 39,390 patients comparing culprit PCI versus MV-PCI in patients with STEMI and MVD during the index procedure were identified finally (Table 1), four randomized $[5,6,7,8]$ and fourteen nonrandomized studies $[9,10,11,12,13,14,15,16,17,18,19,20,21,22]$ published between 2001 and 2013. Nine of the fourteen nonrandomized studies were subanalyses of prospective registries. Details of the screening process for eligible studies are shown in Table S1. Quality assessment results are detailed in Table 2 and Table 3.

\section{Study Characteristics}

Culprit PCI was the more frequently performed PCI strategy (33,594 of 39,390 patients, $85.3 \%$ ). Baseline characteristics of the included studies are presented in Table 4. Compared with the culprit PCI group, patients in the MV-PCI group had a lower rate of diabetes, hypertension and hyperlipidemia. Six studies excluded the patients with cardiogenic shock $[5,6,7,8,14,19]$, and three studies reported the rate of cardiogenic shock [11,16,21]. Ten studies gave information of the use of GP IIb/IIIa inhibitors.

Table 2. Quality of Randomized Studies.

\begin{tabular}{|c|c|c|c|c|c|c|}
\hline $\begin{array}{l}\text { Primary } \\
\text { author }\end{array}$ & $\begin{array}{l}\text { Adequate sequence } \\
\text { generation of allocation }\end{array}$ & $\begin{array}{l}\text { Allocation } \\
\text { concealment }\end{array}$ & $\begin{array}{l}\text { Blinding of participants, } \\
\text { personnel, and outcome } \\
\text { assessors }\end{array}$ & $\begin{array}{l}\text { Complete } \\
\text { outcome data }\end{array}$ & $\begin{array}{l}\text { Free of selective } \\
\text { outcome reporting }\end{array}$ & $\begin{array}{l}\text { Free of other } \\
\text { sources of bias }\end{array}$ \\
\hline Di Mario & Unclear & Unclear & Unclear & Yes & Unclear & Unclear \\
\hline Ochala & Unclear & Unclear & Unclear & Yes & Unclear & Unclear \\
\hline Politi & Yes & Unclear & Unclear & Yes & Unclear & Unclear \\
\hline Wald & Yes & Unclear & Yes & No & Yes & Unclear \\
\hline
\end{tabular}


Table 3. Quality of nonrandomized Studies.

\begin{tabular}{|c|c|c|c|}
\hline Primary author & Control of confounders & Blinded assessment of angiography data & Preferred $\mathrm{PCl}$ strategy \\
\hline Abe & \pm (subanalysis of prospective registry) & - & Operator decision \\
\hline Bauer & \pm (subanalysis of prospective registry) & - & - \\
\hline Carvender & \pm (subanalysis of prospective registry) & - & - \\
\hline Corpus & - & - & Operator decision \\
\hline Dziewierz & \pm (subanalysis of prospective registry) & - & - \\
\hline Hannan & \pm (subanalysis of prospective registry) & - & - \\
\hline Jensen & \pm (subanalysis of prospective registry) & - & Operator decision \\
\hline Khattab & Prospective observational & - & Operator decision \\
\hline Kornowski & \pm (subanalysis of prospective registry) & - & Operator decision \\
\hline Mohamad & - & - & - \\
\hline Qarawani & - & - & Operator decision \\
\hline Roe & - & - & Operator decision \\
\hline Toma & \pm (subanalysis of prospective registry) & - & Operator decision \\
\hline Varani & \pm (subanalysis of prospective registry) & - & Operator decision \\
\hline
\end{tabular}

\section{Main Outcomes}

Short-term mortality was reported in 15 studies including 36,687 patients. In-hospital or 30-day death occurred in 1,515 of 31,349 patients $(4.83 \%)$ who underwent culprit PCI versus 370 of 5,338 patients $(6.93 \%)$ who received MV-PCI. Signs of heterogeneity were found across trials $\left(\mathrm{I}^{2}=70 \%\right)$ and a randomized model was used. Compared with culprit PCI, MV-PCI was associated with an increased short-term mortality (OR: 0.50, 95\% CI: 0.32 to $0.77, \mathrm{p}=0.002)$. Pooled short-term outcome data are detailed in Fig. 1.

Long-term mortality for both strategies was reported in 16 studies including 7,905 patients. There were 362 long-term follow up deaths among 5,670 patients $(6.38 \%)$ who received culprit PCI, whereas 245 deaths occurred among 2,235 (10.96\%) patients who received MV-PCI. Heterogeneity was found across trials $\left(\mathrm{I}^{2}=67 \%\right)$ and a randomized model was used. MV-PCI was associated with an obviously increased long-term mortality in comparison with culprit PCI strategy (OR: 0.52 , 95\% CI: 0.36 to $0.74, \mathrm{p}<0.001)$. Pooled long-term outcome data are illustrated in Fig. 2.

Four studies are available of the short-term renal dysfunction detail. Politi et al. [7] defined renal dysfunction as an increase in serum creatinine values of $0.5 \mathrm{mg} / \mathrm{dl}$ or greater or a $25 \%$ or greater relative increase from baseline within 72 hours following PCI. Abe et al. [9] defined renal dysfunction as an increase in serum creatinine values of $0.5 \mathrm{mg} / \mathrm{dl}$ or greater or a $25 \%$ or greater relative increase from baseline within 1 week following exposure to contrast medium. Cavender et al. [11] defined renal dysfunction as a new requirement for dialysis or an increase in creatinine to $>2 \mathrm{mg} / \mathrm{dl}$ and 2 times the baseline creatinine. Qarawani et al. [19] defined it as a rise of $30 \%$ and more in creatinine within 24 hours from the baseline value. To sum up, renal dysfunction occurred in 503 of 26,131 patients (1.92\%) who underwent culprit PCI versus 93 of 3,348 patients $(2.78 \%)$ who received MV-PCI. No heterogeneity was found among the studies $\left(\mathrm{I}^{2}=0 \%\right)$ and a fixed effects model was used. The difference between two groups are significant (OR: 0.77 , 95\% CI: 0.61 to $0.97, p=0.03$, which indicates that the MV-PCI may increase the risk of renal dysfunction because of the high dose of contrast agent (Fig. 3).
Nine articles reported on long-term reinfarction, 1,449 cases in the culprit PCI group and 847 cases in the MV-PCI group. No heterogeneity was found among the studies $\left(\mathrm{I}^{2}=41 \%\right)$ and a fixed effects model was used. No significant difference was found between the two groups (OR: $1.13,95 \%$ CI: 0.76 to $1.67, \mathrm{p}=0.55$ ) (Fig. 4).

Five studies gave the information of long-term revascularization, 421 cases in the culprit PCI group and 424 cases in the MV-PCI group. Signs of heterogeneity were not found across trials $\left(\mathrm{I}^{2}=46 \%\right)$ and a fixed model was used. MV-PCI was associated with an obviously decreased long-term revascularization in comparison with culprit PCI strategy (OR: 2.65, 95\% CI: 1.80 to $3.90, \mathrm{p}<0.001$ ) (Fig. 5).

\section{Sensitivity Analysis}

We performed sensitivity analyses by repeating analyses following removal of each study, one at a time (data not shown). No single study had excessive influence on the results for primary or secondary endpoints.

The results of randomized trials only and both randomized and nonrandomized trials are different which showed in Table 5. Assessment of funnel plots suggested no publication bias.

\section{Discussion}

Our analysis suggested that MV-PCI strategy is associated with an increased short-term mortality (OR: $0.50,95 \%$ CI: 0.32 to $0.77, \mathrm{p}=0.002$ ), long-term mortality (OR: $0.52,95 \% \mathrm{CI}: 0.36$ to $0.74, \mathrm{p}<0.001$ ), and risk of renal dysfunction (OR: 0.77, 95\% CI: 0.61 to $0.97, \mathrm{p}=0.03$ ) compared with culprit PCI strategy, while it reduced the incidence of revascularization (OR: 2.65, 95\% CI: 1.80 to $3.90, \mathrm{p}<0.001)$. No significant difference was found between the two groups in terms of the rate of reinfarction.

MVD has been proved to be associated with a poor prognosis in STEMI patients. Appropriate management of these patients has always been a topic of debate. Current guidelines recommend that in the absence of hemodynamic compromise, PCI during STEMI should only focus on the culprit lesion. Other lesions are addressed during subsequent elective revascularization. Justifications for these guidelines include [23]: 1) the acute phase of STEMI is a 


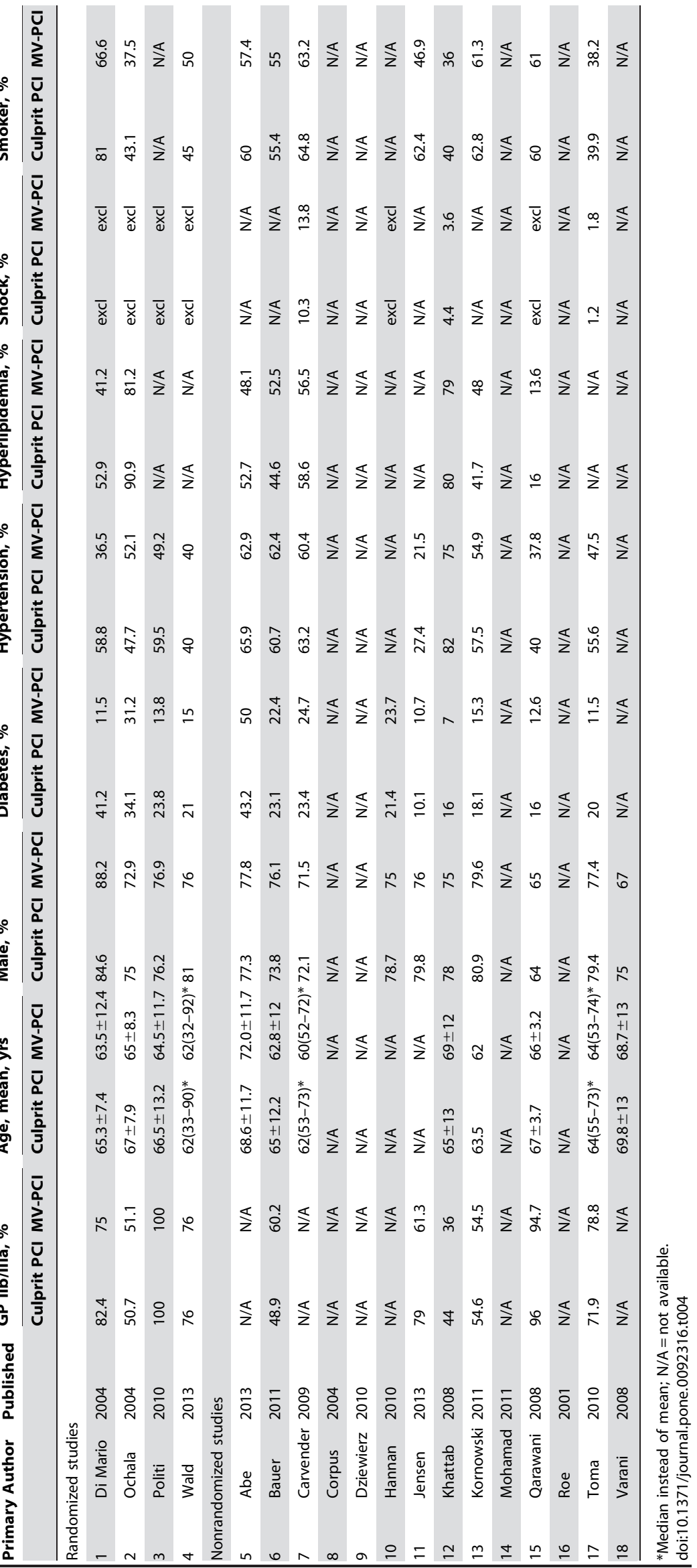




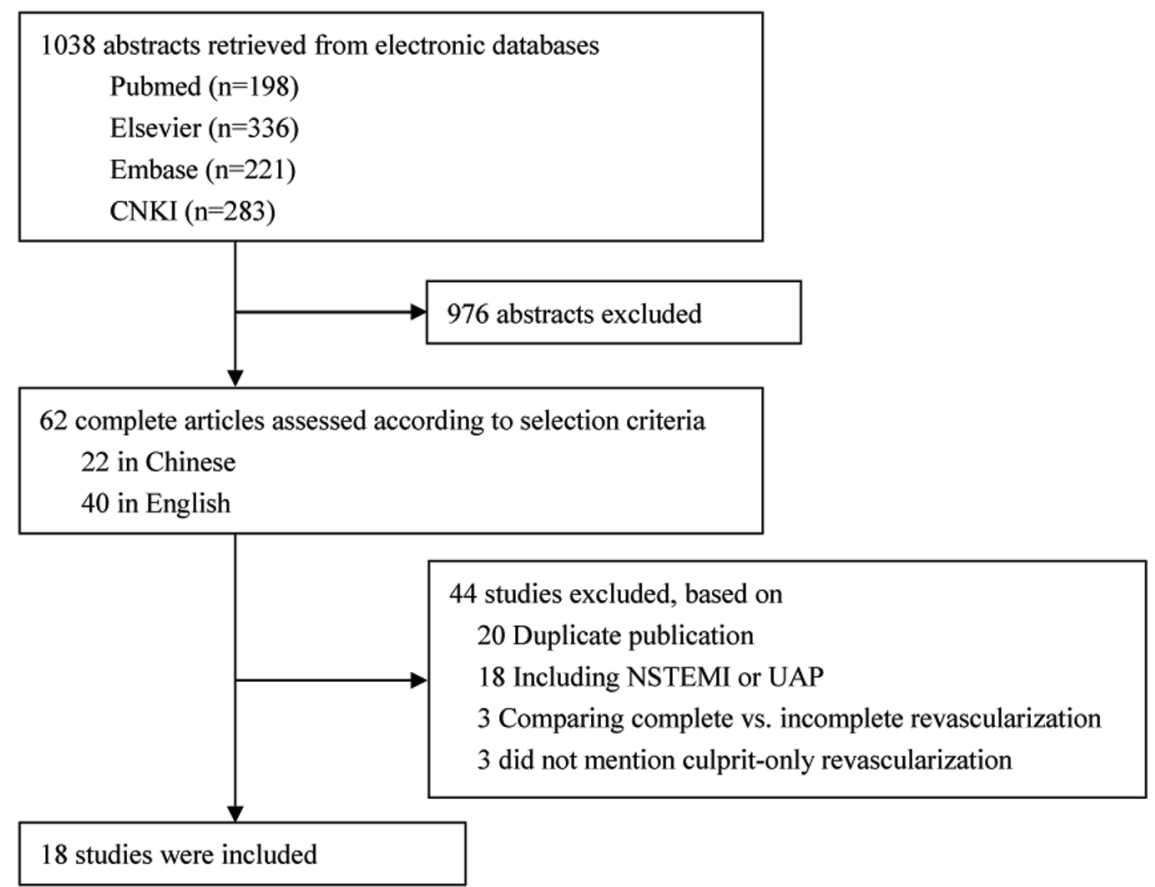

Figure 1. Culprit PCI Versus MV-PCI Short-Term Mortality. doi:10.1371/journal.pone.0092316.g001

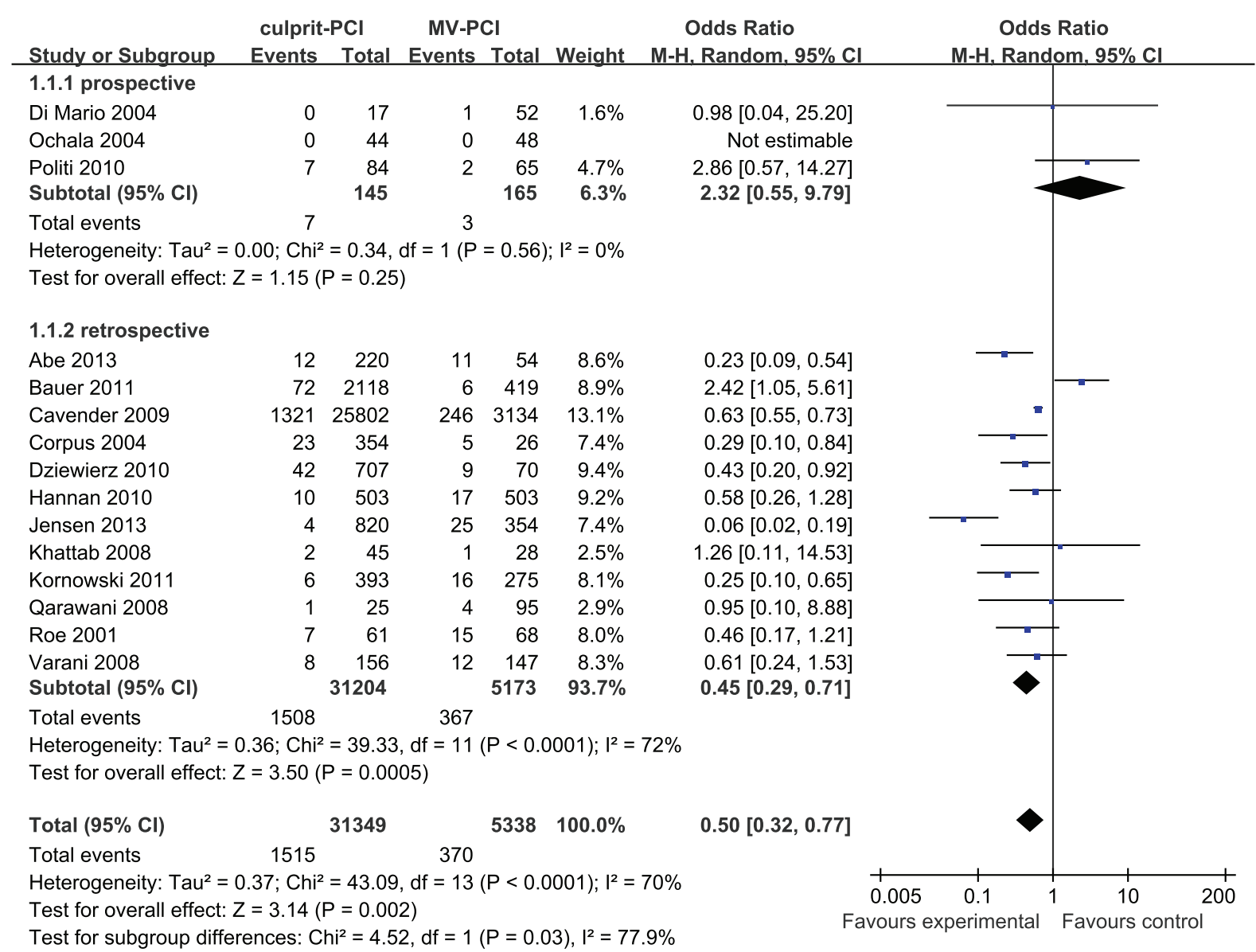

Figure 2. Culprit PCI Versus MV-PCI Long-Term Mortality. doi:10.1371/journal.pone.0092316.g002 


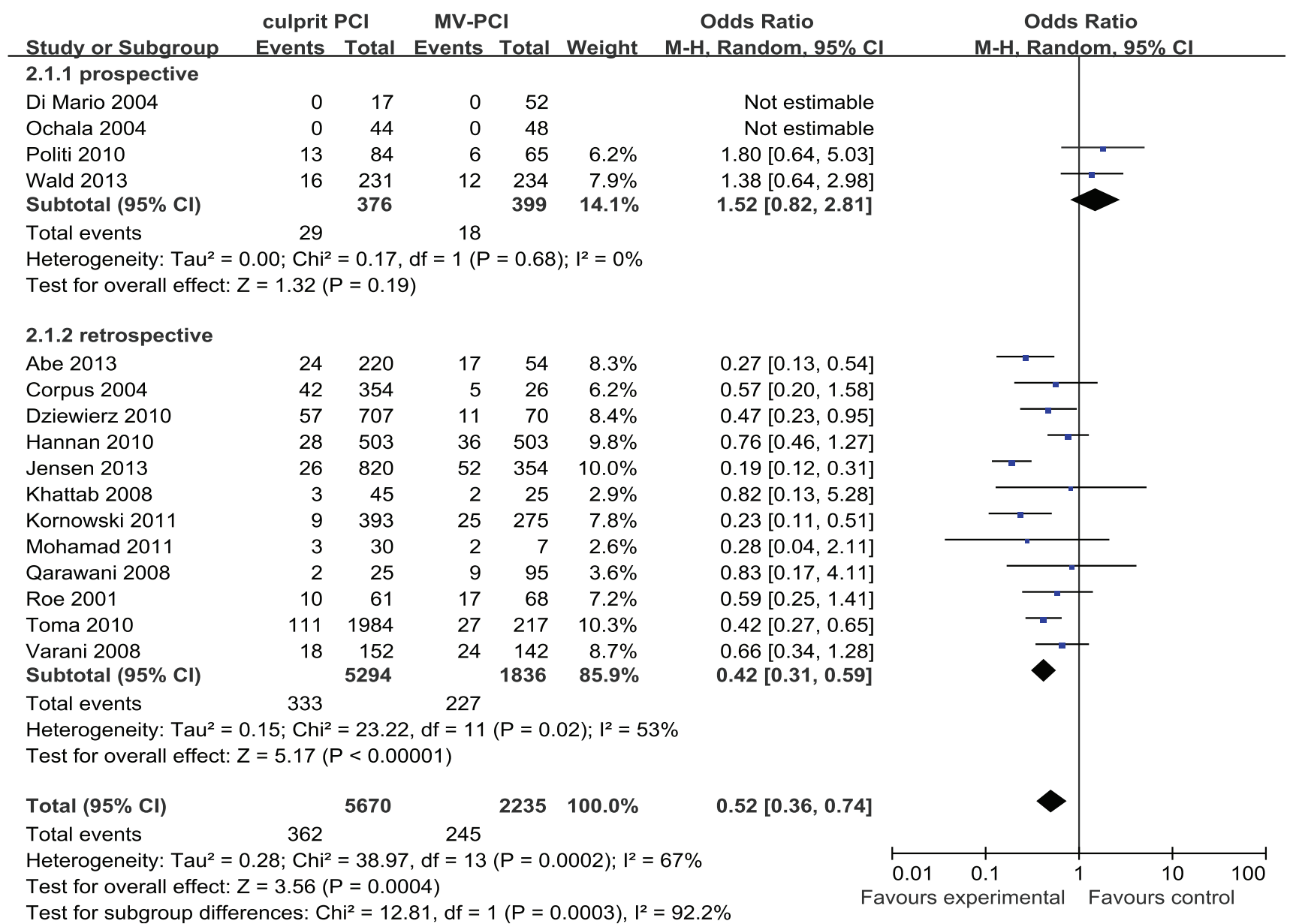

Figure 3. Culprit PCI Versus MV-PCI Renal Dysfunction.

doi:10.1371/journal.pone.0092316.g003

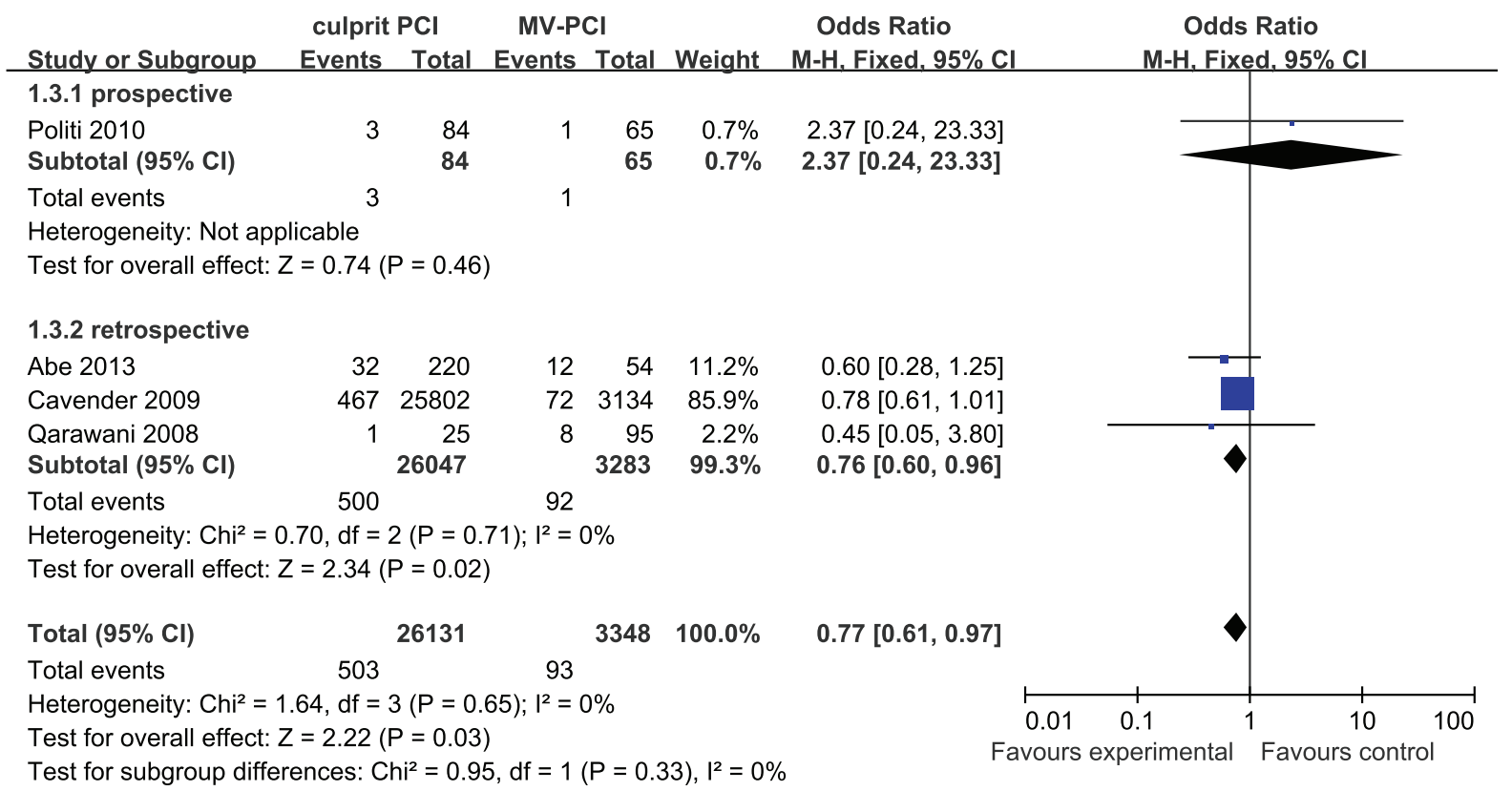

Figure 4. Culprit PCI Versus MV-PCI Long-Term Reinfarction. doi:10.1371/journal.pone.0092316.g004 


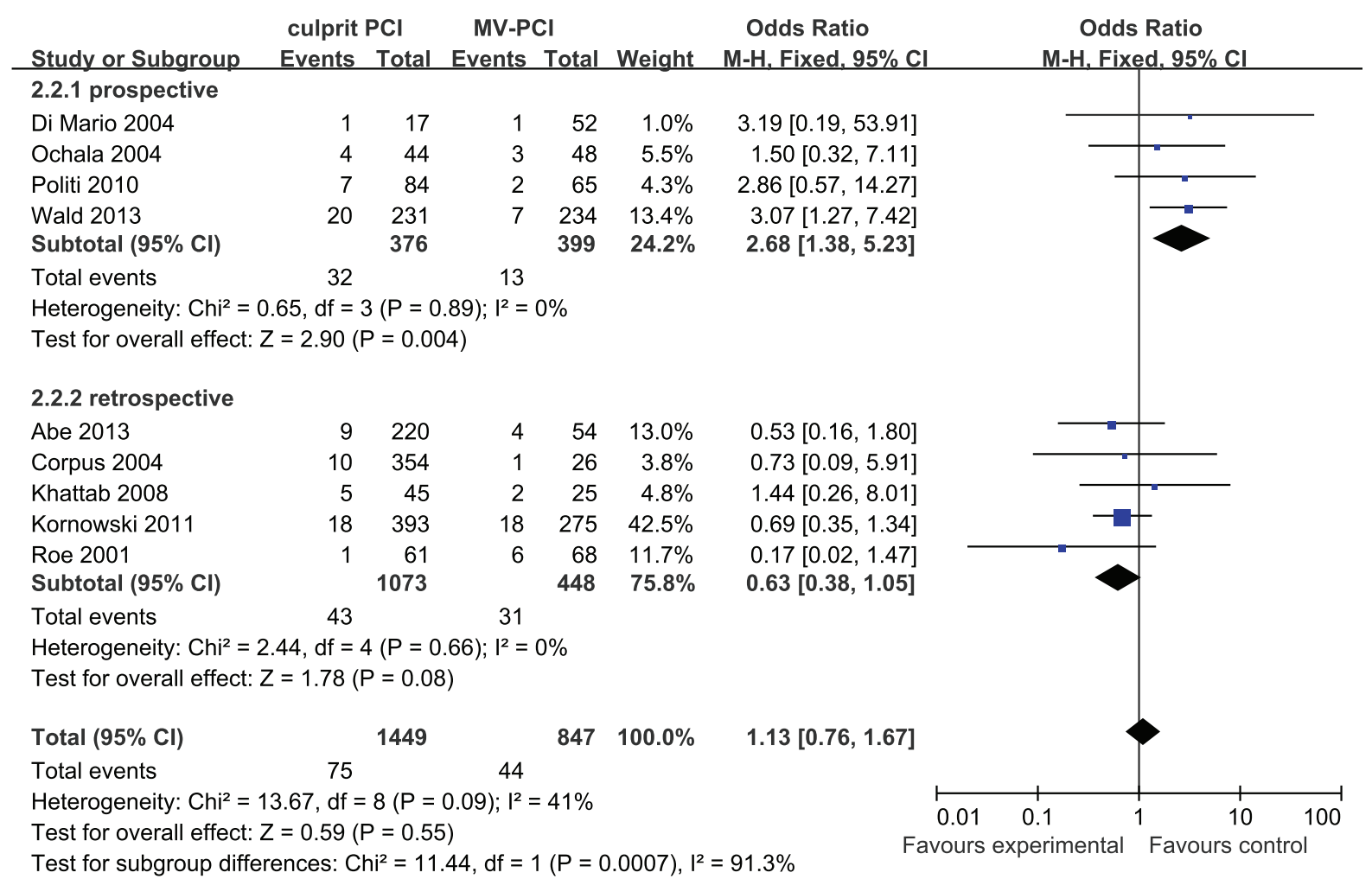

Figure 5. Culprit PCI Versus MV-PCI Long-Term Revascularization. doi:10.1371/journal.pone.0092316.g005

highly unstable condition (haemodynamic instability, heart failure, arrhythmias, resuscitation, and patient stress among others) that does not favor performance of PCI, and additional intervention is probably safer after the patient is stabilized; 2) the acute phase of STEMI is extremely prothrombotic and inflammatory which contributes to a higher risk for additional PCI; 3) diffuse coronary spasms (either due to endothelial dysfunction or due to catecholamine use) are frequently present in the acute phase of STEMI, and this may lead to overestimation of stenosis severity in nonculprit vessels; 4) decisions to perform non-culprit vessel PCI during the acute phase of STEMI are usually not supported by objective evidence for the presence of myocardial ischemia in regions supplied by these non-culprit vessels; 5) MV-PCI increases the radiation dose, contrast overload, and risk of contrast-induced nephropathy. Counter arguments include concerns that plaque instability may be present in large areas of the coronary tree rather than limited to the culprit lesion. Consequently, MV-PCI might achieve complete revascularization by treating secondary unstable lesions and thereby shorten cumulative hospital stays and costs. Patients may also be more comfortable following treatment of all lesions during index hospitalization.

The report was written in accordance to the PRISMAstatement (Checklist S1).Our findings support the current guidelines which indicate that the non-culprit vessel should not be treated during the index procedure. Although analysis of only the four small scaled randomized trials has different even opposite results. It is notable that the largest single-blind, randomized study, called the Preventive Angioplasty in Acute Myocardial Infarction (PRAMI) trail [8], enrolled 465 patients at five centers in the United Kingdom, with 231 assigned to the culprit PCI group and 234 to the MV-PCI group. The recruitment was stopped early after a recommendation from the data and safety monitoring committee that was based on a highly significant difference between groups $(p<0.001)$ in the incidence of the primary outcome favoring MV-PCI. The combined rate of cardiac death, nonfatal myocardial infarction, or refractory angina was reduced

Table 5. Subgroup Analysis of Randomized Trails Compared with Overall Analysis.

\begin{tabular}{lll}
\hline Endpoints & Preferred strategy \\
\cline { 2 - 3 } & Randomized and nonrandomized trails & Randomized trails \\
\hline Short-term mortality & Culprit PCl & Equal \\
Reng-term mortality & Culprit PCl & Equal \\
Reinfarction & Culprit PCl & $\mathrm{MV}-\mathrm{PCl}$ \\
Revascularization & Equal & $\mathrm{MV}-\mathrm{PCl}$ \\
\hline doi:10.1371/journal.pone.0092316.t005 & $\mathrm{MV}-\mathrm{PCl}$ \\
\hline
\end{tabular}


by $65 \%$, and absolute risk reduction of 14 percentage points over 23 months. The findings also suggest that MV-PCI may lead to less ischemia testing after the index procedure. Another randomized trial enrolled only 214 patients, with 84 patients in the culprit PCI group, 65 in the MV-PCI group, and 65 in the staged PCI group [7]. This study showed a significant benefit for MV-PCI, compared to culprit PCI, for long-term major adverse cardiac events (MACE) after a mean follow-up of 2.5 years. The HEpacoat for cuLPrit or multivessel stenting for Acute Myocardial Infarction (HELP AMI) study [5] enrolled only 69 patients, with 17 patients in the culprit PCI group and 52 in the MV-PCI group. In this study, MV-PCI did not significantly increase in-hospital MACE $(0$ and $3.8 \%$ in culprit and MV-PCI groups, respectively, $\mathrm{p}=0.164)$. Revascularization in the culprit PCI group at the 12 month follow-up was not statistically significant (35 vs $17 \%$, $\mathrm{p}=0.247)$. The trial's limitations included unequal randomization and use of heparin-coated stents which may be subject to bias.

A meta-analysis comparing culprit PCI, MV-PCI and staged PCI strategies found that MV-PCI was associated with highest mortality rates at both short- and long-term follow up, in which staged PCI strategy was defined as PCI confined to culprit vessel only, after which lesions in non-culprit vessel were treated during planned secondary procedures [24]. A proper analysis on the secondary endpoints was not possible because data were only available for a minority of the included studies. This meta-analysis included some patients with non-ST-segment elevation myocardial infarction (NSTEMI), and thus their included studies were different from ours. In addition, patients with STEMI or NSTEMI have different treating strategies.

\section{Limitations}

Only four studies were randomized. Consequently, the inclusion of nonrandomized studies introduces a potential selection bias, which means the benefit of culprit PCI shown in Table 5 may simply derive from selection bias towards patients with less severe or more stable coronary artery disease. There is the potential for ascertainment bias due to unequal follow-up.

\section{References}

1. Sorajja P, Gersh BJ, Cox DA, McLaughlin MG, Zimetbaum P, et al. (2007) Impact of multivessel disease on reperfusion success and clinical outcomes in patients undergoing primary percutaneous coronary intervention for acute myocardial infarction. Eur Heart J 28: 1709-1716.

2. Steg PG, James SK, Atar D, Badano LP, Blomstrom-Lundqvist C, et al. (2012) ESC Guidelines for the management of acute myocardial infarction in patients presenting with ST-segment elevation. Eur Heart J 33: 2569-2619.

3. Levine GN, Bates ER, Blankenship JC, Bailey SR, Bittl JA, et al. (2011) 2011 ACCF/AHA/SCAI Guideline for Percutaneous Coronary Intervention. A report of the American College of Cardiology Foundation/American Heart Association Task Force on Practice Guidelines and the Society for Cardiovascular Angiography and Interventions. J Am Coll Cardiol 58: e44-e122.

4. Stroup DF, Berlin JA, Morton SC, Olkin I, Williamson GD, et al. (2000) Metaanalysis of observational studies in epidemiology: a proposal for reporting. Metaanalysis Of Observational Studies in Epidemiology (MOOSE) group. JAMA 283: 2008-2012.

5. Di Mario C, Mara S, Flavio A, Imad S, Antonio M, et al. (2004) Single vs multivessel treatment during primary angioplasty: results of the multicentre randomised HEpacoat for cuLPrit or multivessel stenting for Acute Myocardial Infarction (HELP AMI) Study. Int J Cardiovasc Intervent 6: 128-133.

6. Ochala A, Smolka GA, Wojakowski W, Dudek D, Dziewierz A, et al. (2004) The function of the left ventricle after complete multivessel one-stage percutaneous coronary intervention in patients with acute myocardial infarction. J Invasive Cardiol 16: 699-702

7. Politi L, Sgura F, Rossi R, Monopoli D, Guerri E, et al. (2010) A randomised trial of target-vessel versus multi-vessel revascularisation in ST-elevation myocardial infarction: major adverse cardiac events during long-term followup. Heart 96: 662-667.

8. Wald DS, Morris JK, Wald NJ, Chase AJ, Edwards RJ, et al. (2013) Randomized trial of preventive angioplasty in myocardial infarction. N Engl J Med 369: 1115-1123.
Multiple combinations of angiographic and clinical findings, number of diseased vessels, location and type of occlusions, total chronic occlusions, Killip class, renal function, and other factors vary by individual. This introduces a level of complexity that is best addressed by individualized clinical decision-making.

Further, the operator's intent to perform culprit PGI or MVPCI was not prospectively registered in a majority of the studies and may be influenced by important patient characteristics that we were unable to account for. Staged PCI was allowed for patients in culprit PCI group in some trials which may exaggerate the benefits of culprit PCI. As with many meta-analyses, we did not adjust our analyses for baseline confounders or unmeasured confounders, due to the lack of data in each trial.

\section{Conclusions}

This meta-analysis was based primarily on data derived from nonrandomized studies. It is suggested that culprit PCI is better than MV-PCI procedure in patients with STEMI and MVD. Large-scale randomized trials are urgently needed to further evaluate different revascularization procedures for patients with STEMI and MVD.

\section{Supporting Information}

Table S1 Flow diagram of study inclusion and exclusion.

(DOC)

Checklist S1 PRISMA Checklist of items to include when reporting a systematic review or meta-analysis. (DOG)

\section{Author Contributions}

Conceived and designed the experiments: DFZ XTS SZL. Performed the experiments: DFZ FX MZ WL SY. Analyzed the data: DFZ FX MZ WL SY. Wrote the paper: DFZ XTS FY.

9. Abe D, Sato A, Hoshi T, Takeyasu N, Misaki M, et al. (2013) Initial culprit-only versus initial multivessel percutaneous coronary intervention in patients with STsegment elevation myocardial infarction: results from the Ibaraki Cardiovascular Assessment Study registry. Heart Vessels.

10. Bauer T, Zeymer U, Hochadel M, Mollmann H, Weidinger F, et al. (2013) Prima-vista multi-vessel percutaneous coronary intervention in haemodynamically stable patients with acute coronary syndromes: analysis of over 4.400 patients in the EHS-PCI registry. Int J Cardiol 166: 596-600.

11. Cavender MA, Milford-Beland S, Roe MT, Peterson ED, Weintraub WS, et al. (2009) Prevalence, predictors, and in-hospital outcomes of non-infarct artery intervention during primary percutaneous coronary intervention for ST-segment elevation myocardial infarction (from the National Cardiovascular Data Registry). Am J Cardiol 104: 507-513.

12. Corpus RA, House JA, Marso SP, Grantham JA, Huber KJ, et al. (2004) Multivessel percutaneous coronary intervention in patients with multivessel disease and acute myocardial infarction. Am Heart J 148: 493-500.

13. Dziewierz A, Siudak Z, Rakowski T, Zasada W, Dubiel JS, et al. (2010) Impact of multivessel coronary artery disease and noninfarct-related artery revascularization on outcome of patients with ST-elevation myocardial infarction transferred for primary percutaneous coronary intervention (from the EUROTRANSFER Registry). Am J Cardiol 106: 342-347.

14. Hannan EL, Samadashvili Z, Walford G, Holmes DJ, Jacobs AK, et al. (2010) Culprit vessel percutaneous coronary intervention versus multivessel and staged percutaneous coronary intervention for ST-segment elevation myocardial infarction patients with multivessel disease. JACC Cardiovasc Interv 3: 22-31.

15. Jensen LO, Thayssen P, Farkas DK, Hougaard M, Terkelsen CJ, et al. (2012) Culprit only or multivessel percutaneous coronary interventions in patients with ST-segment elevation myocardial infarction and multivessel disease. EuroIntervention 8: 456-464.

16. Khattab AA, Abdel-Wahab M, Rother C, Liska B, Toelg R, et al. (2008) Multivessel stenting during primary percutaneous coronary intervention for acute myocardial infarction. A single-center experience. Clin Res Cardiol 97: 32-38. 
17. Kornowski R, Mehran R, Dangas G, Nikolsky E, Assali A, et al. (2011) Prognostic impact of staged versus "one-time" multivessel percutaneous intervention in acute myocardial infarction: analysis from the HORIZONSAMI (harmonizing outcomes with revascularization and stents in acute myocardial infarction) trial. J Am Coll Cardiol 58: 704-711.

18. Mohamad T, Bernal JM, Kondur A, Hari P, Nelson K, et al. (2011) Coronary revascularization strategy for ST elevation myocardial infarction with multivessel disease: experience and results at 1-year follow-up. Am J Ther 18: 92-100.

19. Qarawani D, Nahir M, Abboud M, Hazanov Y, Hasin Y (2008) Culprit only versus complete coronary revascularization during primary PCI. Int J Cardiol 123: 288-292.

20. Roe MT, Cura FA, Joski PS, Garcia E, Guetta V, et al. (2001) Initial experience with multivessel percutaneous coronary intervention during mechanical reperfusion for acute myocardial infarction. Am J Cardiol 88: 170-173, A6.
21. Toma M, Buller GE, Westerhout GM, Fu Y, O'Neill WW, et al. (2010) Nonculprit coronary artery percutaneous coronary intervention during acute STsegment elevation myocardial infarction: insights from the APEX-AMI trial. Eur Heart J 31: 1701-1707.

22. Varani E, Balducelli M, Aquilina M, Vecchi G, Hussien MN, et al. (2008) Single or multivessel percutaneous coronary intervention in ST-elevation myocardial infarction patients. Catheter Cardiovasc Interv 72: 927-933.

23. Widimsky P, Holmes DJ (2011) How to treat patients with ST-elevation acute myocardial infarction and multi-vessel disease? Eur Heart J 32: 396-403.

24. Vlaar PJ, Mahmoud KD, Holmes DJ, van Valkenhoef G, Hillege HL, et al (2011) Culprit vessel only versus multivessel and staged percutaneous coronary intervention for multivessel disease in patients presenting with ST-segment elevation myocardial infarction: a pairwise and network meta-analysis. J Am Coll Cardiol 58: 692-703. 